\title{
REDUÇÃO DO TEMPO DE TRANSIÇÃO DE UMA UNIDADE DE DESTILAÇÃO OPERANDO COM AÇÃO DE CONTROLE DISTRIBUÍDA ENTRE ESTÁGIOS DO ESGOTAMENTO E DA RETIFICAÇÃO
}

\author{
G. N. MELLO ${ }^{1}$, R. A. F. MACHADO ${ }^{2}$, C. MARANGONI ${ }^{3}$ \\ ${ }^{1}$ Universidade da Região de Joinville, Mestrado em Engenharia de Processos \\ ${ }^{2}$ Universidade Federal de Santa Catarina, Depto de Engenharia Química e Engenharia de Alimentos \\ ${ }^{3}$ Universidade Federal de Santa Catarina, Campus Blumenau \\ E-mail para contato: cintia.marangoni@ufsc.br
}

\begin{abstract}
RESUMO - Baseado na proposta do uso de aquecimento distribuído para controle de colunas de destilação visando à minimização de transientes, neste trabalho testou-se um sistema com ação de aquecimento distribuída entre o calor cedido no refervedor e em um prato da seção de esgotamento, seguido de um com ação distribuída de resfriamento usando a vazão de refluxo e o calor retirado em um prato da seção de retificação e comparou-se com um sistema convencional de controle dual de temperatura. Utilizando o software Hysys ${ }^{\circ}$, foram avaliados efeitos de perturbações na temperatura de alimentação variando de $\pm 2^{\circ} \mathrm{C}$ até $\pm 20^{\circ} \mathrm{C}$. Observou-se maior redução no tempo de transição para a malha de controle de temperatura do último estágio com perturbações acima de $14^{\circ} \mathrm{C}$ para ambos os casos distribuídos. Assim, pode-se concluir que a abordagem de controle com ação distribuída permite a redução de transientes tanto quando a ação necessária é de aquecimento ou resfriamento.
\end{abstract}

\section{INTRODUÇÃO}

Uma vez que colunas de destilação podem ser consideradas um dos equipamentos mais importantes dentro de uma indústria, constituindo a grande fração do investimento fixo e dos custos operacionais para fornecer os produtos finais dentro das especificações adequadas, várias técnicas de controle são estudadas (Szabó et al., 2012). Os sistemas para controlar variáveis em colunas em destilação são desenvolvidos com os mais variados objetivos, tais como: aumentar a produção, minimizar transientes de operação assim como o consumo energético e produtos fora de especificação, reduzir os custos envolvidos no processo, eliminar riscos inerentes a este, e melhorar a qualidade dos produtos finais.

$\mathrm{O}$ rápido alcance do estado estacionário minimiza o tempo necessário para atender às especificações desejadas do produto quando o sistema é perturbado. Entretanto, inúmeras dificuldades intrínsecas ao processo e existentes no controle destas unidades são o fator responsável pela geração de longos transientes. $\mathrm{O}$ resultado que se observa é que a coluna opera por um determinado tempo fora do seu equilíbrio, mesmo empregando-se sistemas de controle bem ajustados. Este 


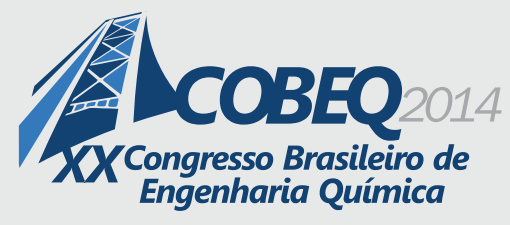

19 a 22 de outubro de 2014

Florianópolis/SC

comportamento é decorrente da configuração da coluna em estágios, necessitando de ações sucessivas de aquecimento e resfriamento para ocorrer a transferência de calor e massa nos estágios e, portanto necessita-se da propagação da ação de controle por toda a unidade para minimizar as perturbações e manter os padrões de qualidade estabelecidos para os produtos.

A solução mais abordada para reduzir o tempo de operação fora da condição desejada consiste na implantação de técnicas de controle, mais especificamente as que consideram a dinâmica da unidade em sua estrutura. Conforme citado por Huyck et al. (2014), nos últimos 40 anos a técnica de controle preditivo baseado em modelo (MPC) tem sido a mais aplicada. Ainda, a grande maioria das unidades operam com apenas uma malha de controle de qualidade de forma que ambas as composições das correntes de base e topo são mantidas em limites aceitáveis. O emprego de duas malhas de controle para manter rigorosamente qualidade destas duas correntes impõe dificuldades devido a complexidade de implementação e de acoplamento existente no processo (Fruehauf e Mahoney, 1993). Por este motivo, tais sistemas tendem a serem difíceis de serem implementados, e industrialmente controladores simples, como PID (proporcional-integral-derivativo) ainda são largamente empregados.

Outra proposta que vem sendo utilizada para redução de transiente consiste na mudança da condição de operação convencional da coluna. Modificações em sua estrutura física podem melhorar o desempenho do processo e, por consequência, a qualidade dos produtos de interesse. Dentre estas opções, podem-se citar as unidades intensificadas energeticamente, mais especificamente as diabáticas (Jana, 2010), que operam com o fornecimento de calor do refervedor distribuído nos estágios da seção de esgotamento e a retirada deste nos pratos da seção de retificação.

Aliando este conceito de distribuição de calor nos pratos com o objetivo de minimizar transientes de operação e a aplicação de controladores clássicos (PID), uma proposta de controle com ação distribuída foi proposta e avaliada por Marangoni (2005). Para isso, foi construída uma unidade piloto prevendo a utilização apenas de pontos de aquecimento nos pratos da coluna visando testar experimentalmente a técnica proposta. Posteriormente, Werle (2007) avaliou dentro da mesma estratégia, diferentes condições de operação bem como o consumo energético, novamente obtendo resultados positivos para a abordagem proposta. Os autores citados observaram que a introdução de aquecimento distribuído ao longo da coluna mostrou-se como uma opção válida para a redução de transientes, possibilitando uma dinâmica mais rápida e menores volumes processados fora dos parâmetros de qualidade pré-definidos, consequentemente, obtendo um menor consumo energético com reprocessamento. No entanto, em todos os testes realizados, somente pontos de aquecimento na seção de esgotamento da unidade foram considerados (Marangoni e Machado, 2007; Werle et al., 2009; Marangoni et al. 2013). Conforme já citado, estudos de destilação diabática demonstram que, do ponto de vista energético, deve-se distribuir tanto o fornecimento de calor do refervedor como a retirada pelo condensador (Johannessen e Røsjorde, 2007). Devido ao caráter experimental da unidade construída, a avaliação de retirada de calor fazendo-se uso de pratos da unidade não pode ser avaliada. Experimentalmente, o calor cedido aos pratos da seção de esgotamento foi realizado por meio da inserção de resistências elétricas nos pratos da unidade. Porém, para operar com o resfriamento, seria necessário, por exemplo, a concepção de um estágio em que uma serpentina (interna ou superficial) com um fluido de resfriamento pudesse proporcionar tal ação. 
Desta forma, a configuração proposta neste trabalho dá continuidade de forma simulada a este tema. Para tanto, a unidade experimental avaliada nos estudos de Marangoni (2005) e Werle (2007) foi simulada, validando-se os testes com controle convencional. $\mathrm{O}$ objetivo foi avaliar a abordagem operacional utilizando aquecimento e resfriamento nos pratos de uma coluna de destilação para fins de controle, buscando a redução do tempo de transição quando a unidade foi perturbada na temperatura de alimentação variando de $\pm 2{ }^{\circ} \mathrm{C}$ até $\pm 20^{\circ} \mathrm{C}$.

\section{MATERIAIS E MÉTODOS}

O presente estudo de simulação está baseado na unidade experimental contínua apresentada em Marangoni (2005) e que possui 13 pratos perfurados, sendo o refervedor o estágio zero e o tanque acumulador, o 14. A alimentação é realizada no prato 4. Nos ensaios experimentais, a corrente de alimentação foi introduzida acima do quarto prato da unidade (o refervedor representa o estágio zero). Devido a estas características da planta, é necessário realizar a alimentação no simulador no quinto prato de forma a reproduzir os resultados dos ensaios reais (Oliveira, 2013).Detalhes construtivos são apresentados em Marangoni et al. (2004). Na Figura 1 é ilustrada a coluna com as correntes do processo.

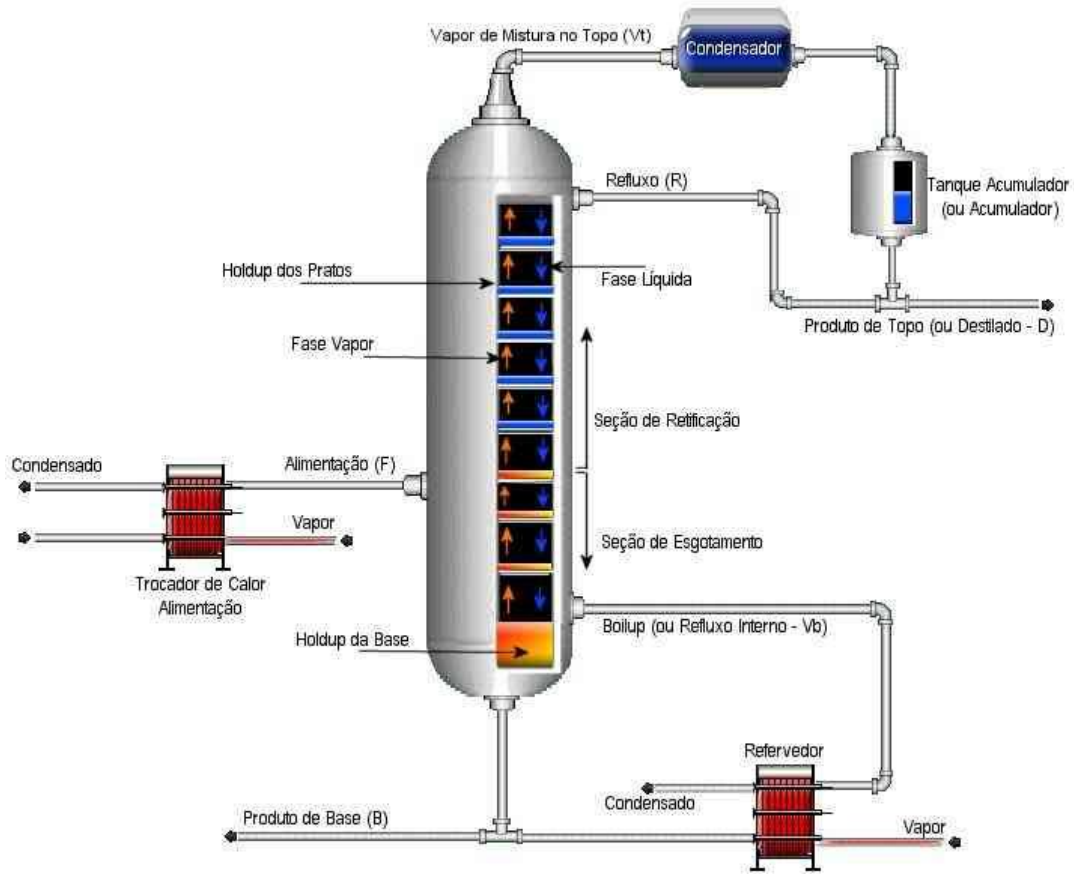

Figura 1 - Ilustração dos equipamentos e correntes da coluna de destilação simulada (Fonte: Marangoni, 2005).

As simulações foram realizadas utilizando-se o software Hysys® da AspenTech, versão 7.3, com a mistura etanol e água e definindo-se como pacote termodinâmico o UNIQUAC (Universal Quasi-Chemical Theory). As condições utilizadas são apresentadas na Tabela 1. 
Tabela 1 - Condições operacionais e parâmetros da coluna de destilação.

\begin{tabular}{|l|c|}
\hline \multicolumn{1}{|c|}{ Variável } & Valor \\
\hline Temperatura de alimentação & subresfriada $\left(\approx 80^{\circ} \mathrm{C}\right)$ \\
\hline Vazão volumétrica de alimentação & $300 \mathrm{~L} / \mathrm{h}$ \\
\hline Fração volumétrica de etanol na alimentação & 0,2 \\
\hline Pressão no topo da coluna & $1,2 \mathrm{bar}$ \\
\hline Queda da pressão ao longo da coluna & $0,15 \mathrm{bar}$ \\
\hline Razão de refluxo & 6 \\
\hline
\end{tabular}

Duas estratégias diferentes de controle foram avaliadas: a convencional e a distribuída onde controladores do tipo PID foram implementados nas seguintes malhas para o sistema denominado como convencional: (1) Controle da temperatura da base, manipulando-se a carga térmica fornecida no refervedor e (2) Controle da temperatura do último estágio, manipulando-se a vazão de refluxo. A abordagem distribuída consistiu na utilização destas malhas, acrescida de outra intermediária que foi acionada separadamente: Controle da temperatura do prato 3 ou do prato 11 manipulando-se o fornecimento/retirada de calor em cada um destes estágios. A definição pelo uso do estágio foi realizada a partir de estudos de análise de sensibilidade (Mello et al., 2013). A avaliação foi realizada de forma separada, onde perturbações na temperatura da alimentação variando de -2 a $-20^{\circ} \mathrm{C}$ foram testadas com a estratégia distribuída utilizando o prato 3 , e perturbações variando de +2 a $+20^{\circ} \mathrm{C}$ foram testadas com o uso do prato 11.

Os parâmetros utilizados nos controladores são apresentados na Tabela 2 e foram determinados a partir de uma sintonia fina com base nos valores utilizados experimentalmente. As malhas de controle das temperaturas do refervedor e do prato 13 foram ajustadas considerando o acoplamento existente, e sintonizadas com o auxílio de desacopladores estáticos. Estes valores foram mantidos tanto no controle com ação distribuída quanto no convencional. As malhas de controle de temperatura dos pratos 3 e 11 foram consideradas descentralizadas.

Tabela 2 - Parâmetros dos controladores PID utilizados.

\begin{tabular}{|l|c|c|c|}
\hline Parâmetro & Refervedor & Prato 13 & Prato 3 e 11 \\
\hline $\mathrm{K}_{\mathrm{c}}$ & $3,90^{*}$ & $0,70^{* *}$ & $7,22^{*}$ \\
\hline$\tau_{\mathrm{i}}(\mathrm{s})$ & $7,45 \times 10^{-2}$ & $9,67 \times 10^{-2}$ & $2,57 \times 10^{-2}$ \\
\hline$\tau_{\mathrm{d}}(\mathrm{s})$ & $9,17 \times 10^{-3}$ & $1,00 \times 10^{-2}$ & $5,33 \times 10^{-3}$ \\
\hline
\end{tabular}

É importante ressaltar que do ponto de vista experimental, inserções e retiradas de calor devem ser promovidas com o auxílio de dispositivos acoplados aos pratos da unidade para permitir tal ação. Nas simulações, o software permite que apenas uma simples corrente de energia seja inserida ao estágio e definida em termos de troca de calor. Para tanto, definiu-se a faixa de 0 a $3,5 \mathrm{~kW}$ (aplicada experimentalmente), onde $100 \%$ corresponde a zero de retirada de calor (valor mínimo), e $0 \%$ significa que o valor total de resfriamento foi aplicado (neste caso, $-3,5 \mathrm{~kW}$ ). 


\section{RESULTADOS}

Na Figura 2(a) são apresentados os resultados obtidos para a derivada da temperatura do refervedor ao longo do tempo quando uma perturbação realizada na temperatura da alimentação de $-14^{\circ} \mathrm{C}$, comparando-se o sistema convencional e o distribuído utilizando o prato 3 . A análise da derivada foi definida para determinação do tempo de transição pois esta indica que cada desvio do valor nulo corresponde a um desvio do estado estacionário. Mesmos resultados são apresentados na Figura 2(b) para a temperatura do prato 13.
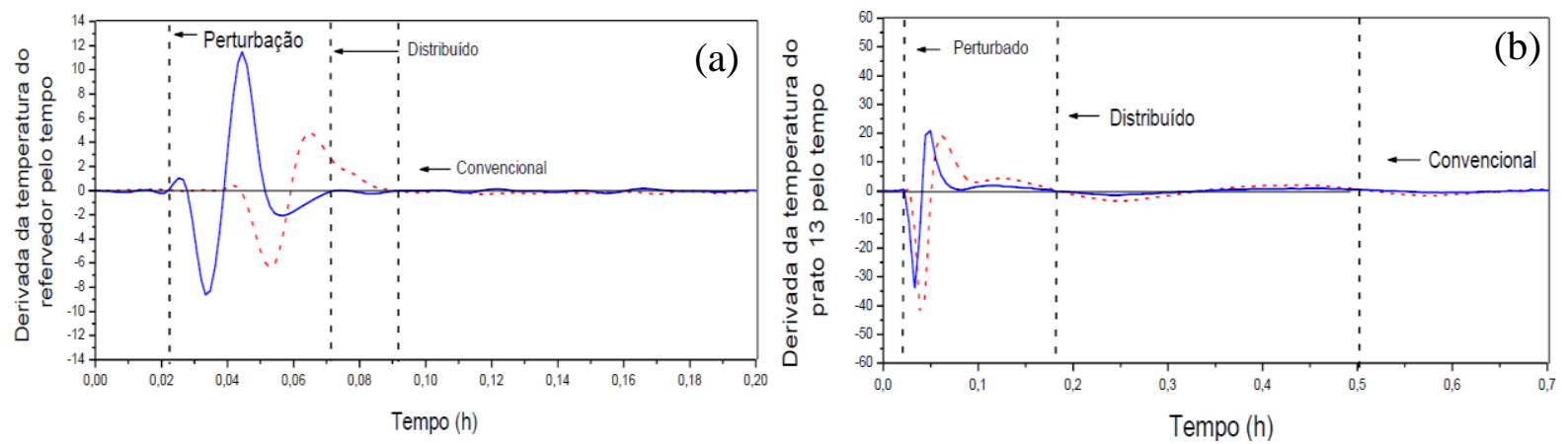

Figura 2 - Derivada da temperatura do refervedor (a) e do prato 13 (b) em relação ao tempo para

a perturbação de $-14{ }^{\circ} \mathrm{C}$ na temperatura de alimentação comparando-se as estratégias convencional (- - ) e distribuída aplicado no prato 3 (-) em relação ao valor do set point (-).

A análise da figura permite verificar que para a temperatura do refervedor, o tempo de transição observado com a abordagem convencional foi de $0,09 \mathrm{~h}$ (5,4 minutos), enquanto na abordagem distribuída foi de 0,07 h (4,2 minutos), obtendo-se uma redução neste valor maior que $22 \%$. Já para a malha de controle de temperatura do prato 13, o tempo de transição para a abordagem convencional foi de $0,5 \mathrm{~h}$ (30 minutos) e para a abordagem distribuída de $0,18 \mathrm{~h}(10,8$ minutos), obtendo-se uma redução de aproximadamente 64\%. Ressalta-se que a oscilação observada não se trata de problema numérico e sim característica do processo, o qual possui uma resposta oscilatória amortecida (típica de uma coluna de destilação).

$\mathrm{Na}$ Figura 3 são apresentados os resultados para perturbações de $+14^{\circ} \mathrm{C}$, quando o controle distribuído foi utilizado acionando-se o prato 11 . Neste caso, o tempo de transição para a malha de controle da temperatura do refervedor foi de $0,10 \mathrm{~h}$ (6 minutos) para a estratégia convencional e 0,08 h (4,8 minutos) para a estratégia distribuída, ou seja, uma redução de $20 \%$. Para a malha de controle da temperatura do prato 13 verificou-se que tempo de transição para a estratégia convencional foi de 0,44 h (26,4 minutos) e para a estratégia distribuída de 0,12 h $(7,2$ minutos), obtendo-se uma redução de $72,72 \%$.

Estes resultados são refletidos na quantidade de produto gerado fora da especificação, conforme ilustrado na Tabela 3. Observa-se que tanto quando ações de aquecimento quanto ações de resfriamento são exigidas, a proposta de controle distribuído permite que o sistema retorne mais rapidamente ao estado estacionário, reduzindo o tempo de transição e por consequência, o volume de produto gerado fora da especificação. 


\section{9 a 22 de outubro de 2014 \\ Florianópolis/SC}
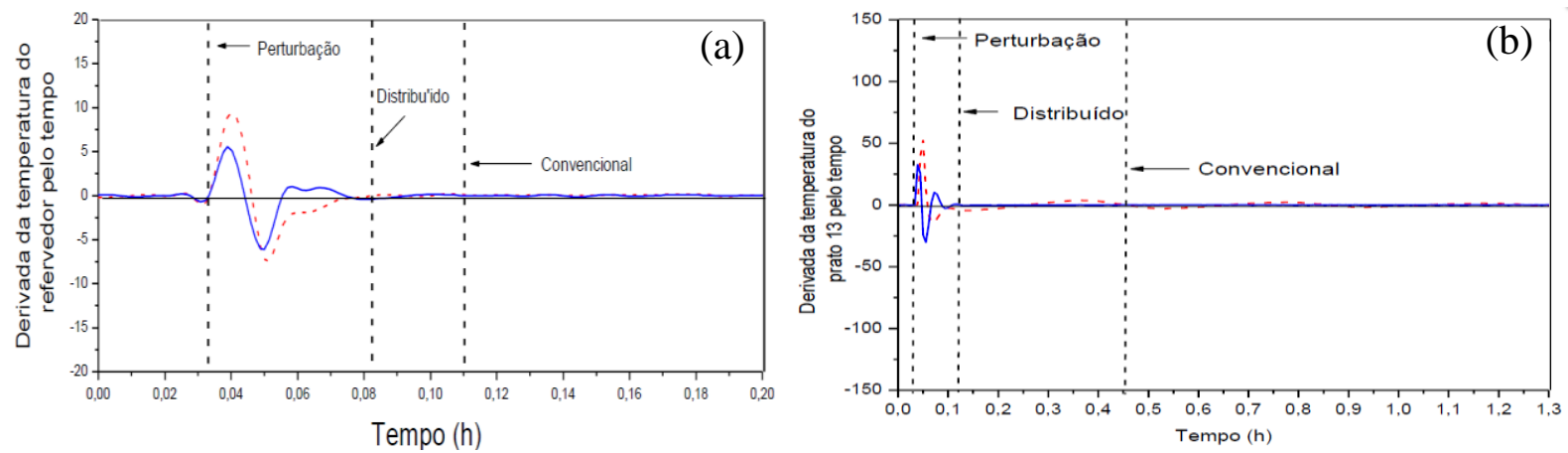

Figura 2 - Derivada da temperatura do refervedor (a) e do prato 13 (b) em relação ao tempo para a perturbação de $+14{ }^{\circ} \mathrm{C}$ na temperatura de alimentação comparando-se as estratégias convencional (- - -) e distribuída aplicado no prato 11 (-) em relação ao valor do set point (-).

Tabela 3 - Quantidade de produto fora do especificado (em volume) da corrente de destilado durante o tempo de transição para as estratégias convencional e distribuída.

\begin{tabular}{|l|c|c|c|}
\hline Variável & Estratégia convencional & $\begin{array}{c}\text { Estratégia distribuída } \\
\text { usando o prato } 3\end{array}$ & $\begin{array}{c}\text { Estratégia distribuída } \\
\text { usando o prato } 11\end{array}$ \\
\hline Vazão $\left(\mathrm{m}^{3} / \mathrm{h}\right)$ & $4,267 \times 10^{-5}$ & $2,087 \times 10^{-5}$ & $1,95 \times 10^{-6}$ \\
\hline
\end{tabular}

Diferentes perturbações foram realizadas na temperatura de alimentação e o tempo de transição foi determinado para as variáveis já analisadas (temperatura do refervedor e temperatura do prato 13). Primeiramente foi analisado o controle distribuído na região de esgotamento da coluna, com o controle e fornecimento de calor do prato 3 e em seguida para o controle distribuído utilizando o prato 11 para perturbações de $\pm 2,8$ e $20^{\circ} \mathrm{C}$, e os resultados são apresentados na Tabela 4 .

Observa-se uma redução significativa no tempo de transição para perturbação de $20^{\circ} \mathrm{C}$. Para as perturbações inferiores não foi verificado diferenças expressivas no tempo de transição quando comparada as duas estratégias testadas. Atribui-se este resultado ao impacto da perturbação que é pequeno nestes casos. É importante ressaltar que em nenhum caso a estratégia distribuída apresentou desempenho inferior a convencional.

Um resultado que deve ser evidenciado é o de que quando perturbações positivas foram realizadas, a estratégia distribuída que utiliza o prato 3 não demonstra ganhos em relação a convencional. Este fato é explicado em virtude do tipo de perturbação aplicada, que exige o resfriamento da unidade e, portanto o prato 3 não é acionado. Quando perturbações negativas foram realizadas na temperatura da alimentação, o perfil de temperatura da unidade é conduzido a valores inferiores ao do estado estacionário, e a ação de aquecimento é necessária. Porém, a o contrário do verificado com perturbações positivas, neste caso, o controle distribuído utilizando o prato 11 demonstrou ganhos no tempo de transição por meio de ações de resfriamento (que a princípio não seriam necessárias). A contribuição desta ação pode ser verificada principalmente na redução do transiente da temperatura do prato 13. 
Tabela 4 - Tempo de transição das malhas de controle de temperaturas do refervedor e do prato 13 quando aplicada a estratégia convencional e distribuída frente a diferentes perturbações na temperatura de alimentação.

\begin{tabular}{|c|c|c|c|c|}
\hline Temperatura & $\begin{array}{c}\text { Estratégia } \\
\text { convencional (h) }\end{array}$ & $\begin{array}{l}\text { Estratégia distribuída } \\
\text { com prato } 3(\mathrm{~h})\end{array}$ & $\begin{array}{c}\text { Estratégia } \\
\text { convencional (h) }\end{array}$ & $\begin{array}{l}\text { Estratégia distribuída } \\
\text { com prato } 11(\mathrm{~h})\end{array}$ \\
\hline \multicolumn{5}{|c|}{ Perturbação $-2^{\circ} \mathrm{C}$} \\
\hline Refervedor & 0,11 & 0,05 & 0,1 & 0,06 \\
\hline Estágio 13 & 0,35 & 0,09 & 0,39 & 0,09 \\
\hline \multicolumn{5}{|c|}{ Perturbação $+2{ }^{\circ} \mathrm{C}$} \\
\hline Refervedor & 0,07 & 0,07 & 0,08 & 0,08 \\
\hline Estágio 13 & 0,22 & 0,22 & 0,33 & 0,14 \\
\hline \multicolumn{5}{|c|}{ Perturbação $-8^{\circ} \mathrm{C}$} \\
\hline Refervedor & 0,08 & 0,08 & 0,08 & 0,08 \\
\hline Estágio 13 & 0,37 & 0,18 & 0,33 & 0,14 \\
\hline \multicolumn{5}{|c|}{ Perturbação $+8^{\circ} \mathrm{C}$} \\
\hline Refervedor & 0,08 & 0,08 & 0,06 & 0,06 \\
\hline Estágio 13 & 0,23 & 0,23 & 0,47 & 0,15 \\
\hline \multicolumn{5}{|c|}{ Perturbação $-20^{\circ} \mathrm{C}$} \\
\hline Refervedor & 0,06 & 0,001 & 0,08 & 0,06 \\
\hline Estágio 13 & 0,48 & 0,001 & 0,35 & 0,11 \\
\hline \multicolumn{5}{|c|}{ Perturbação $+20^{\circ} \mathrm{C}$} \\
\hline Refervedor & 0,08 & 0,07 & 0,09 & 0,001 \\
\hline Estágio 13 & 0,06 & 0,05 & 0,47 & 0,001 \\
\hline
\end{tabular}

\section{CONCLUSÕES}

Neste trabalho avaliou-se a proposta de uma estratégia de controle de colunas de destilação com ação distribuída e observou-se que esta abordagem mantém a unidade operando mais próxima dos valores de referência produzindo menor quantidade de produto fora do especificado tanto quando pratos da seção de esgotamento quanto retificação são utilizados. Desta forma, verificou-se que a estratégia é eficiente tanto quanto perturbações exigem ações de aquecimento quanto de resfriamento da unidade. Os resultados foram mais evidentes para perturbações de maior magnitude.

É importante ressaltar que certamente um desempenho melhor teria sido obtido caso tivesse sido utilizado uma sintonia específica para cada abordagem de controle (convencional e distribuída). Porém, isso prejudicaria a comparação uma vez que o objetivo do trabalho consistiu em verificar os benefícios de uma abordagem distribuída e não a comparação entre diferentes abordagens de sintonia de controladores.

\section{REFERENCIAS}

FRUEHAUF, P. S.; MAHONEY, D. P. Distillation column control design using steady state models: usefulness and limitations. ISA Trans., v. 32, p. 157-175, 1993. 
HUYCK, B.; de BRABANTER, J.; de MOOR, B.; van IMPE J. F.; LOGIST, F. Online model predictive control of industrial processes using low level control hardware: A pilot-scale distillation column case study. Int. J. Refrig., v. 30, p. 499-505, 2007.

JANA, A.K.; Heat integrated distillation operation, Appl. Energy, v. 87, p. 1477-1494, 2010.

JOHANNESSEN, E.; RØSJORDE, A. Equipartition of entropy production as an approximation to the state of minimum entropy production in diabatic distillation, Energy, v. 32, p. 467-473, 2007.

MARANGONI, C.; Implementação de uma estratégia de controle com ação distribuída em uma coluna de destilação, (Doutorado em Engenharia Química) Universidade Federal de Santa Catarina, Florianópolis, 2005.

MARANGONI, C.; MACHADO, R. A. F. Distillation Tower with Distributed Control Strategy: Feed Temperature Loads, Chem. Eng. Technol., v. 30, p. 1292-1297, 2007.

MARANGONI, C.; MACHADO, R. A. F.; BOLZAN, A. Distributed heat supply for distillation control to reduce feed composition disturbance effects. Chem. Eng. Technol.. v. 36, p. 2071-2079, 2013.

MARANGONI, C.; PASETTI, G.; RICO, J. E. N.; MACHADO, R. A. F.; BOLZAN, A. Construção e Instrumentação com Tecnologia Fieldbus de uma Coluna Piloto de Destilação, Revista Petroquímica: v. XXVIII, p. 55-59, 2004.

MELLO, G. N.; MACHADO, R. A. F.; MARANGONI, C. Análise de Sensibilidade de uma Unidade de Destilação Fracionada para Implementação de Controle com Ação Distribuída. 11 Congreso Interamericano de Computación Aplicada a la Industria de Procesos - CAIP'2013, Lima, Peru. v. 1, p. 1-10, 2013.

OLIVEIRA, D. L.; Desenvolvimento de um simulador para o estudo de estratégias de controle em colunas de destilação com aquecimento distribuído, (Mestrado em Engenharia Química), Universidade Federal de Santa Catarina, Florianópolis, 2013.

SZABÓ, L.; NÉMETH, S.; SZEIFERT, F. Three-level control of a distillation column. Engineering, v. 4, p. 675-681, 2012.

WERLE, L.O.; Minimização dos Transientes através do Aquecimento Distribuído em uma Coluna de Destilação. (Mestrado em Engenharia Química) - Departamento de Engenharia Química, Universidade Federal de Santa Catarina, Florianópolis, 2007.

WERLE, L. O.; MARANGONI, C.; TELEKEN, J. G.; SAYER, C.; MACHADO, R. A. F. Control Strategy with Distributed Action for Minimization of Transients in Distillation Column. Comput.Aided Chem. Eng., v. 27, p. 1527-1532, 2009. 